\title{
An Empirical Model for Durations in Stocks
}

\author{
Ola Simonsen* \\ Department of Economics, Umeå University \\ SE-901 87 Umeå, Sweden
}

\begin{abstract}
This paper considers an extension of the univariate autoregressive conditional duration model to which durations from a second stock are added. The model is empirically used to study durations in two traded stocks, Ericsson B and AstraZeneca, on the Stockholm Stock Exchange. It is found that including durations from a second stock may add explanatory power to the univariate model. Ericsson B is Granger causing durations in AstraZeneca, while AstraZeneca is not Granger causing durations in Ericsson B. Volume, spread and trade intensity changes have significant effects for both series.
\end{abstract}

Key Words: Finance, multivariate, duration, transaction data, market microstructure.

JEL Classification: C12, C32, C41, G14.

${ }^{*}$ The author would like to thank Kurt Brännäs and Jörgen Hellström for valuable comments and suggestions. Financial support from the Wallander Foundation B is gratefully acknowledged. 


\section{Introduction}

This paper empirically examines the dependence between durations in stocks traded on the Stockholm Stock Exchange. In this context durations are the times between two consecutive transactions in a stock. To empirically capture dependence between durations in stocks the Autoregressive Conditional Duration model (ACD) of Engle and Russell (1998) is extended by adding lagged durations from a second stock to the conditional mean function.

The reason for studying the time between transactions in a stock is that it may provide insights on how information enters the market (Easly and O'Hara, 1992). Short durations between transactions in a stock are indicative of new information to the stock market. If new information becomes available it may not only be stock specific but may also be sector specific or even affect all stocks on the stock market. Accordingly, new information may lead to dependence between durations in different stocks.

With the availability of ultra high frequency data (i.e., within the day series where every transaction is registered) standard econometric tools may not be appropriate as transactions are irregularly spaced over time. One way of handling the irregularity is to aggregate data into regularly spaced intervals and apply, e.g., count data models. However, aggregation results in information loss which is not always desirable. Albeit the complication of modelling irregularly spaced data one influential approach for handling the data is the ACD model. It models the duration between transactions and conditions the duration on recent durations and other explanatory variables. Within the ACD framework several extensions and applications of the original Engle and Russell (1998) model have been presented (e.g., Bauwens and Giot, 2000; Dufour and Engle, 2000).

Models for the dependence between durations in more than one duration series have proven rather complicated, e.g., Engle and Lunde (2003), Russell (1999) and Bauwens and Hautsch (2004). The difficulty is in modelling an expected duration while at the same time accounting for events during the duration. Pairs of durations with the same starting time would be ideal, but are rarely available in transaction data. Both Engle and Lunde (2003) and Russell (1999) advance a model for the dependence between the trade and the quote arrival processes. Bauwens and Hautsch (2004) present a model for the dependence between durations and report results for German stocks. The model of Engle and Lunde (2003) is closely related to the ACD model as it treats one of the duration series as censored and applies the ACD model. Russell (1999) and Bauwens and Hautsch (2004) take another approach and instead model the intensities. An application of the model of Russell (1999) for dependences between duration series have been given by Spierdijk, Nijman and van Soest (2002). They find positive dependence between stocks 
active in the same industry sector.

Here, a technically simpler approach to capturing dependence between duration series is suggested. In the ACD model durations from a second stock beyond the focused one are added to the conditional mean function and the result is empirically evaluated. Admittedly the proposed models are rather ad hoc even though they attempt to capture the main features of the data generating process. However, the empirical evidence of the current type of models may serve a purpose or inspiration for both building a base of empirical evidence and more probabilistic work.

Maximum likelihood and conditional least square are suggested as estimators of the unknown parameters. Further, an impulse response function is plotted and Granger causality is studied. Empirical results are presented for 26 trading days in Ericsson B and AstraZeneca at the Stockholm Stock Exchange.

The outline of the paper is the following. In Section 2 we give a brief account of the ACD model and present the extended version. The section ends with details concerning estimation. Section 3 presents the data, while Section 4 presents the empirical results. The final section concludes.

\section{Model and estimation}

In this section we first give a brief account of the ACD model and then proceed to discuss the extension which is aimed at accounting for a second transaction series. The estimation of unknown parameters is also considered.

\subsection{Autoregressive conditional duration model}

In the ACD model the time between consecutive transactions is modelled. The duration, $d_{i}$, is the time between two consecutive transactions at $t_{i-1}$ and $t_{i}$, i.e. $d_{i}=t_{i}-t_{i-1}$. The conditional expectation of a duration $d_{i}$ is specified as $E\left(d_{i} \mid d_{i-1}, \ldots, d_{1} ; x\right)=\theta_{i}$. Where $d_{i}$, is conditioned on past durations and other explanatory variables $x$, and $\theta_{i}$ is specified in such a way that $\epsilon_{i}=d_{i} / \theta_{i}$ is independent and identically distributed. The $\theta_{i}$ may be parameterized as

$$
\theta_{i}=\omega+\sum_{j=1}^{p} \alpha_{j} d_{i-j}+\sum_{j=1}^{q} \beta_{j} \theta_{i-j}+\pi^{\prime} x_{i-1} .
$$

In (1) $\theta_{i}$ is parameterized with $p$ lagged durations and $q$ lags of conditional durations. This is called an $\operatorname{ACD}(p, q, x)$ where $p$ and $q$ are the orders of the lags in the mean function and $x_{i}$ is a vector of explanatory variables such as volume and spread at time $i$. 
The unconditional mean and variance of an $\operatorname{ACD}(1,1)$ are easy to obtain. By assuming that $\epsilon_{i}$ follows a standard exponential distribution, $\pi=0$ in (1), and that $d_{i}$ is weakly stationary the mean is

$$
E\left(d_{i}\right)=\mu=\frac{\omega}{1-\alpha-\beta} .
$$

The time invariant unconditional mean together with the obvious $d_{i}>0$ condition imply the restrictions $\omega>0$ and $\alpha+\beta<1$. The unconditional variance of the $\operatorname{ACD}(1,1)$ is given by

$$
\operatorname{Var}\left(d_{i}\right)=\sigma^{2}=\mu^{2} \times \frac{1-\beta^{2}-2 \alpha \beta}{1-\beta^{2}-2 \alpha \beta-2 \alpha^{2}} .
$$

For finite variance $\beta^{2}+2 \alpha \beta+2 \alpha^{2}<1$ must hold.

\section{$2.2 \quad$ Extended ACD model}

To capture the dependence between durations in stocks we suggest that the ACD model be extended by adding durations from stocks beyond the focused one. The available durations for the focused stock are past finished durations in the same and the other stocks, and the length of the most recent incomplete duration in other stocks. For the presentation of the model we introduce a counting process and then proceed with the suggested formulation of the model.

Let the number of events for the $k^{t h}$ duration series by time $t$ be denoted by $N^{k}(t)$ and $t_{0}^{k}<t_{1}^{k}<t_{2}^{k}<\ldots<t_{i}^{k}$ the transaction times for the $k^{t h}$ series. The most recent transaction time is then at $t_{N^{k}(t)}^{k}$ and the most recent duration is $d_{N^{k}(t)}^{k}=t_{N^{k}(t)}^{k}-t_{N^{k}(t)-1}^{k}$.

An example with two stocks, 1 and 2, is presented in Figure 1. The expected length of the next duration $d_{N^{1}(t)}^{1}$ is conditioned at $t_{N^{1}(t)-1}^{1}$ in stock 1 . The $d_{N^{1}(t)}^{1}$ may be conditioned on its own past durations, e.g., $d_{N^{1}(t)-1}^{1}, d_{N^{1}(t)-2}^{1}, \ldots, d_{N^{1}(t)-q}^{1}$, and other explanatory variables. If durations from a second stock are added to the conditional mean function, e.g., $d_{N^{2}(t)}^{2}, d_{N^{2}(t)-1}^{2}$ and $d_{N^{2}(t)-2}^{2}$, the most recent added duration $d_{N^{2}(t)}^{2}$ in stock 2 may not be completed when the conditioning takes place in stock 1 . The observed length of the most recent duration $d_{N^{2}(t)}^{2}$ in stock 2 is $\tau_{1}$ where $\tau_{1}=t_{N^{1}(t)-1}^{1}-t_{N^{2}(t)-1}^{2}$ or $\tau_{1}=\sum_{k=1}^{N^{1}(t)} d_{k-1}^{1}-\sum_{k=1}^{N^{2}(t)} d_{k-1}^{2}$, and $\tau_{1} \geq 0$.

The proposed, extended ACD model is in a bivariate case for stocks 1 and 2:

$$
\theta_{N^{1}(t)}^{1}=\omega^{1}+\sum_{j=1}^{p_{1}} \alpha_{j}^{1} d_{N^{1}(t)-j}^{1}+\sum_{j=1}^{q_{1}} \beta_{j^{1}}^{1} \theta_{N^{1}(t)-j}^{1}+\pi^{\prime} x_{N^{1}(t)-1}^{1}+e^{\delta_{0}^{1} \tau_{1}}\left(1+\sum_{j=1}^{r_{1}} \delta_{j}^{1} d_{N^{2}(t)-j}^{2}\right)
$$




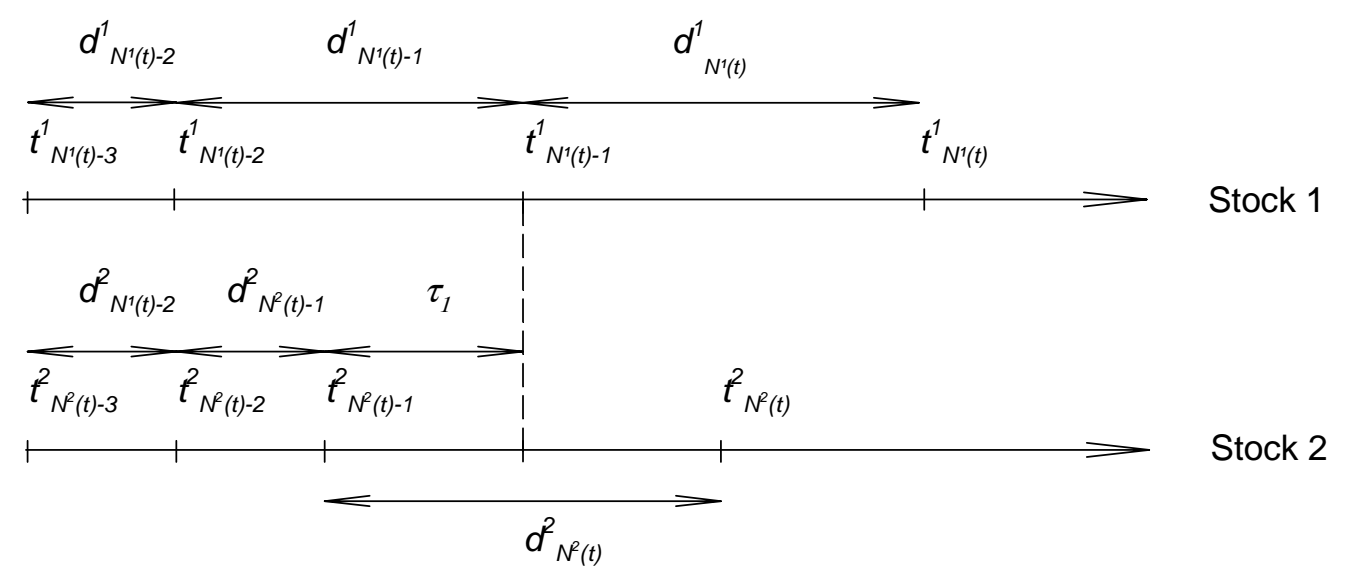

Figure 1: Illustration of two stocks, stock 1 and stock 2, with transaction times $t$ and durations d. $\tau_{1}$ is the observed length of the most recent duration in stock 2 at time $t_{N^{1}(t)-1}^{1}$.

$$
\theta_{N^{2}(t)}^{2}=\omega^{2}+\sum_{j=1}^{p 2} \alpha_{j}^{2} d_{N^{2}(t)-j}^{2}+\sum_{j=1}^{q_{2}} \beta_{j}^{2} \theta_{N^{2}(t)-j}^{2}+\pi^{\prime} x_{N^{2}(t)-1}^{2}+e^{\delta_{0}^{2} \tau_{2}}\left(1+\sum_{j=1}^{r_{2}} \delta_{j^{2}}^{2} d_{N^{1}(t)-j}^{1}\right)
$$

In (4) where completed durations from a stock 2 are added with $r_{1}$ lags, completed durations are given weights dependent on the size of $\tau_{1}$, i.e. depending on how far away in time the completed durations are. The intuition behind the parameterization is that a large value of $\tau_{1}$ in $\exp \left(\delta_{0}^{1} \tau_{1}\right)$ gives low weight to the finished durations from stock 2 and a small value of $\tau_{1}$ gives a larger weight to the finished durations when $\delta_{0}^{1}<0$. The conditional mean function (5) for stock 2 with durations from stock 1 added is formulated in a analogous manner.

When durations from a second stock are added to the conditional mean function as suggested the crucial point is the updating of the mean function. For the most recent duration from stock 2 to enter the mean function (4) a transaction in stock 1 must occur. During a long period without a transaction in stock 1 but with transactions in stock 2 information from stock 2 may not be included in the mean function for stock 1 .

Obviously, the specifications of the final terms in (4)-(5) could take on other forms. For instance, the final term could be additive with $\theta_{N^{2}(t)-j}^{2}, j \geqslant 1$, and $\tau$, entering (4) in a linear way and vice versa.

The conditional moment functions in (4)-(5) are easy to interpret with respect to effects of changes in post durations etc. Obtaining the unconditional mean and variance is, however, quite difficult as it involves taking the expectation of the nonlinear final term in (4)-(5). To illustrate, 
consider the properties of an $\operatorname{ACD}(1,1)$ with one lag of the added duration. The model can be written

$$
d_{N^{1}(t)}^{1}=\theta_{N^{1}(t)}^{1} \epsilon_{N^{1}(t)}^{1}, \quad \theta_{N^{1}(t)}^{1}=\omega^{1}+\alpha^{1} d_{N^{1}(t)-1}^{1}+\beta^{1} \theta_{N^{1}(t)-1}^{1}+e^{\delta_{0}^{1} \tau_{1}}\left(1+\delta_{1}^{1} d_{N^{2}(t)-1}^{2}\right),
$$

where $\epsilon_{N^{1}(t)}^{1}$ follows a standard exponential distribution. By taking expectations of both sides of (6), assuming that $d_{N^{1}(t)}^{1}$ and $\theta_{N^{1}(t)}^{1}$ are weakly stationary the unconditional mean may be expressed as

$$
\mu_{1}=E\left(\theta_{N^{1}(t)}^{1}\right)=E\left(d_{N^{1}(t)}^{1}\right)=\frac{\omega^{1}+\varphi^{1}}{1-\alpha^{1}-\beta^{1}}
$$

where $\varphi^{1}=E\left[e^{\delta_{0}^{1} \tau_{1}}\left(1+\delta_{1}^{1} \theta_{N^{2}(t)}^{2}\right)\right]$ can be either positive or negative depending on the sign of $\delta_{1}^{1}$. To have positive durations the most natural conditions are $\alpha^{1}+\beta^{1}<1$ and $\omega^{1}>-\varphi^{1}$. Obtaining an explicit formulation of $\varphi^{1}$ and the variance appears more difficult then illuminating, and hence detailed expressions are not given.

One useful extension of the original ACD model that ensures positive expected durations is the log-ACD model by Bauwens and Giot (2000). The conditional mean function for a log$\operatorname{ACD}(p, q)$ with explanatory variables is then

$$
\theta_{N^{1}(t)}^{1}=\exp \left[\omega^{1}+\sum_{j=1}^{p_{1}} \alpha_{j}^{1} d_{N^{1}(t)-j}^{1}+\sum_{j=1}^{q_{1}} \beta_{j}^{1} \ln \theta_{N^{1}(t)-j}^{1}+\pi^{\prime} x^{1}+e^{\delta_{0}^{1} \tau_{1}}\left(1+\sum_{j=1}^{r_{1}} \delta_{j^{1}}^{1} d_{N^{2}(t)-j}^{2}\right)\right]
$$

For the specification $\log -\mathrm{ACD}(1,1)$ the only parameter restriction is $\beta^{1}<1$ and $\delta_{0}^{1}<0$.

\subsection{Estimation}

Engle and Russell (1998) popularized the quasi maximum likelihood (QML) estimator building on the exponential distribution for the estimation of the unknown parameters of the ACD model. By extending the conditioning set to also include observed durations in the other duration sequence, the same QML estimator can be used for our purpose. The estimator maximizes the log-likelihood function,

$$
\ln \ell=-\sum_{j=1}^{T} \ln \left[\theta_{j}^{k}+\frac{d_{j}^{k}}{\theta_{j}^{k}}\right] .
$$

The QML estimator is consistent when the conditional mean function is correctly specified. A correctly specified distribution may, however, render a more efficient estimator. For the practical estimation of the parameters we employ the log-ACD specification in (7), which we implement 
in the RATS package and the BFGS algorithm. The standard errors of the parameter estimates are the robust standard errors given by RATS.

Other estimators for the unknown parameters may also be considered, e.g., conditional least squares (CLS) or generalized method of moments (GMM). With these estimators the distribution assumption on the conditional duration is relaxed, though, relaxing the distributional assumption is not a major drawback given that the density is in the exponential family and $\theta_{j}^{k}$ is correctly specified (Bauwens, Giot, Grammig and Veredas, 2004).

Starting with the CLS estimator the prediction error from (4)-(5) is

$$
e_{N^{k}(t)}^{k}=d_{N^{k}(t)}^{k}-\theta_{N^{k}(t)}^{k}
$$

The CLS estimator of the parameters in the vector $\psi^{\prime}$ minimizes the sum of the squared prediction errors

$$
Q(\psi)=e^{\prime} e
$$

where $e^{\prime}=\left(e_{1}^{\prime}, e_{2}^{\prime}\right)$. Standard errors of the estimated parameters are obtained from the robust error option in RATS. A major benefit of leaving the QML based on a univariate exponential model is that joint estimation of the two conditional mean functions is feasible. By this it is easier to extend previous conditional mean functions to, e.g., be functions of lagged conditional means from other duration sequences. Naturally if considering the joint estimation of the conditional mean functions the irregular intervals and updating of the conditional mean functions must be carefully considered.

The GMM estimator introduced by Hansen (1982) may also be considered for our purpose. By utilizing the orthogonality conditions suggested by Grammig and Wellner (2002) and by potentially adding a parametrization for the covariation between duration sequences.

\subsection{Data and descriptive statistics}

The transaction data were downloaded from Ecovision, a provider of real time financial information from the Stockholm Stock Exchange. Two stocks, Ericsson B and AstraZeneca, were recorded for 26 trading days (November 5-December 11, 2002). The companies are active in different lines of business as AstraZeneca is a health care company and Ericsson B is a information technology company. The stocks are two of the most traded stocks on the Stockholm Stock Exchange, albeit the number of transactions in Ericsson B is almost tenfold that of AstraZeneca. Also the average turnover per day in the stocks indicates that Ericsson B is roughly sevenfold more traded than AstraZeneca. AstraZeneca has a turnover of approximately 380 million SEK and Ericsson B 2500 million SEK per day. 
Table 1: Summary statistics for AstraZeneca and Ericsson B durations.

\begin{tabular}{lrrrrrr}
\hline \hline & Mean & Std. & Nr obs & LB $_{100}$ & Min & Max \\
\hline AstraZeneca & 44.6 & 75.4 & 16938 & 6350.5 & 0 & 1403 \\
Ericsson B & 5.2 & 8.0 & 150044 & 149356.9 & 0 & 249 \\
\hline Note: LB 100 & is the Ljung-Box autocorrelation statistic for \\
100 lags.
\end{tabular}

Every transaction is recorded on a second scale with associated volume, bid, ask, and price variables. The tick size, i.e. the minimum amount a price can move, is for AstraZeneca 0.5 SEK and for Ericsson B 0.05 SEK.

Table 1 presents descriptive statistics for the durations between transaction times in the two stocks. The number of observations indicates that Ericsson B is more frequently traded than AstraZeneca. The Ljung-Box statistics are high for both AstraZeneca and Ericsson B. Figure 2 shows the autocorrelation functions for the two series. The autocorrelations are quite small but the decay is quite slow.
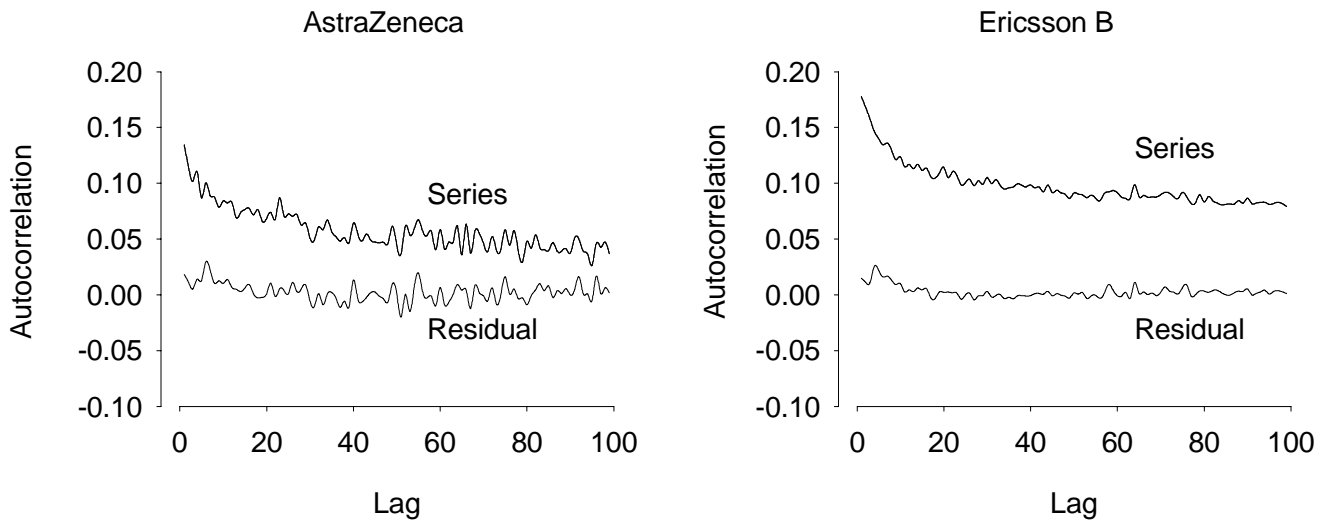

Figure 2: Autocorrelation functions for durations in AstraZeneca and Ericsson B and for the residuals of Models 4 of Tables 2 and 3. Residuals are calculated as $\left(d_{N^{k}(t)}^{k}-\widehat{\theta}_{N^{k}(t)}^{k}\right) \widehat{\theta}_{N^{k}(t)}^{k}$.

Transaction data often show strong intraday seasonality patterns (e.g., Bauwens, Galli and Giot, 2002; Engle and Russell, 1998). Figure 3 shows the intraday seasonality pattern for the duration of the current sample. Durations tend to be shorter when the market opens and near the closing time for both AstraZeneca and Ericsson B. Similar patterns are also present in variables such as volume, spread and trade intensity. To account for potential seasonality of the data the adjusted duration (Engle and Russell, 1998) is computed as $d_{i}=D_{i} / \phi_{i}$, where $D_{i}$ is 


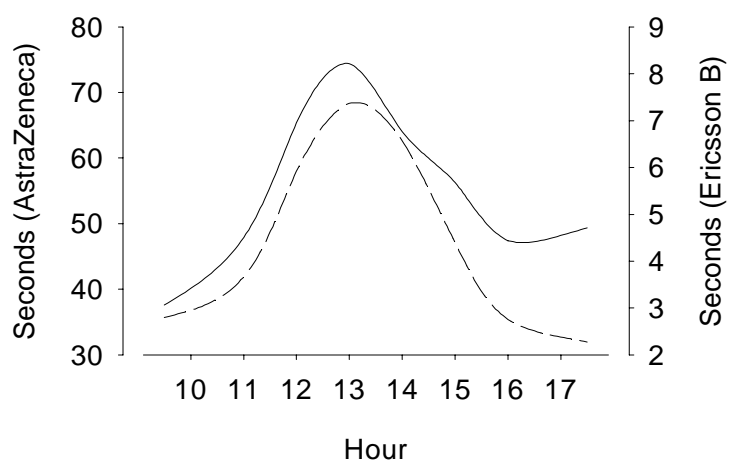

Figure 3: Average duration lengths for AstraZeneca (dashed line) and Ericsson B (solid line) per hour smoothed with a cubic spline.

the duration from the dataset and $\phi_{i}$ is a cubic spline with nodes on each hour. The adjusted duration $d_{i}$ is used for estimation. In a similar way the other variables, volume, spread and trade intensity are adjusted to account for the diurnal effect.

There are many consecutive zero durations, i.e. multiple transactions within a second. To account for all marks within a second we assume that the distance between transactions within a second are equally spaced (cf. Darolles and Gourièroux, 2000).

In the empirical part volume and spread changes are used as explanatory variables together with a measure of trade intensity. A variable capturing the intensity of trade (Engle and Russell, 1998) can be constructed by dividing the number of transactions within a price duration with the length of a price duration. Bauwens and Giot (2000) use a similar approach using instead the spread duration. A price duration is calculated as the time it takes for the price to move a predetermined number of ticks. Obviously a new price duration is initiated more often with a low predetermined tick size. The number of ticks is here chosen as 3. Other studies, e.g., Engle and Russell (1998) suggests the number of ticks as 4. The price used for the price duration is defined as the midprice, i.e. the price in the middle of bid and ask at time $i$.

\section{Results}

The results to be reported are based on the log-ACD model. A main reason for using the logACD in favour of the ACD model is that numerical problems were faced when a pure ACD model were implemented. To find a lag structure we employ the AIC criterion. The minimized AIC gives us the model specification, $\log -\operatorname{ACD}(5,1)$ with two lags of durations from Ericsson $\mathrm{B}$ 
for AstraZeneca, and log- $\mathrm{ACD}(4,1)$ with two lags of durations from AstraZeneca for Ericsson B.

Tables 2-3 give the CLS and QML estimation results for Ericsson B and AstraZeneca with differences in volume, spread, trade intensity and durations as explanatory variables. The parameter estimates for the explanatory variables volume, spread and trade intensity change in Models 3 and 4 are all significant with the QML estimator. The parameter sign for changes in volume and trade intensity are negative for both Ericsson B and AstraZeneca, while spread change has a positive sign for AstraZeneca and a negative one for Ericsson B. For a positive parameter sign a positive change in a variable prolongs the next duration. Brännäs and Simonsen (2003) who also utilize change variables when studying Ericsson B, for a different sample, find negative sign of the spread change but positive one for the volume change.

The estimated parameters $\alpha$ and $\beta$ in Table 3-4 are all significant except for the third and fourth lags of $\alpha$ in estimates of AstraZeneca. The sign of the estimates of the first lagged durations are throughout positive. The following lags have negative sign except for the fourth lag of AstraZeneca. The parameter $\beta$ is near one for both stocks which is also what is found in other studies of transaction data (e.g., Brännäs and Simonsen, 2002). The models estimated without the insignificant parameters were also considered. However, the exclusion results in an AIC that is not the minimum for the models.

The parameters $\delta_{0}, \delta_{1}$ and $\delta_{2}$ from the added dependence term in (4) and (5) are not significant neither for Ericsson B with AstraZeneca nor for AstraZeneca with Ericsson B. However, the signs of the parameters are what may be expected. For both Ericsson B and AstraZeneca $\delta_{0}$ is negative except for Ericsson B with AstraZeneca estimated with CLS. This implies that the impact of the other stocks is decreasing with the size of $\tau$. The parameters $\delta_{1}$ and $\delta_{2}$ are positive except for the second lag in Table 2, Model 2 and CLS for Ericsson B with AstraZeneca. A positive sign implies that long durations in the added duration series has a positive impact on the conditional mean function.

When considering the CLS estimator the parameter signs are the same and roughly the same size although with a few exceptions. Some of the estimates turn insignificant when using the CLS, e.g., trade intensity for both Ericsson B and AstraZeneca and the second lagged duration of AstraZeneca. The serial correlation of the residuals is still high for Ericsson B and is increased for AstraZeneca.

One may use a Granger causality test (Granger, 1969) to examine if the inclusion of a duration for another stock to the conditional mean in (4) and (5) adds explanatory power or not. A likelihood ratio test is applied to test if the individual parameters, $\delta_{0}, \delta_{1}$ and $\delta_{2}$ are jointly, significantly different from zero (cf. Tables 2-3). The test statistic has a $\chi^{2}$ distribution in large samples and a 5 percent critical value of 7.82 . It is found that AstraZeneca is not 
Table 2: Estimates of Ericsson B models with explanatory variables and durations from AstraZeneca (robust $t$ statistics in parenthesis).

\begin{tabular}{|c|c|c|c|c|c|}
\hline Variable & Model 1 & Model 2 & Model 3 & Model 4 & 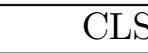 \\
\hline \multirow[t]{2}{*}{$d_{t-1}$} & 0.098 & 0.098 & 0.096 & 0.096 & 0.0378 \\
\hline & $(34.5)$ & $(42.6)$ & $(39.6)$ & $(42.19)$ & $(40.1)$ \\
\hline \multirow[t]{2}{*}{$d_{t-2}$} & -0.013 & -0.013 & -0.013 & -0.013 & -0.004 \\
\hline & $(-3.3)$ & $(-4.3)$ & $(-3.4)$ & $(-4.1)$ & $(-10.6)$ \\
\hline \multirow[t]{2}{*}{$d_{t-3}$} & -0.014 & -0.014 & -0.013 & -0.013 & -0.004 \\
\hline & $(-3.6)$ & $(-4.2)$ & $(-3.4)$ & $(-3.7)$ & $(-37.3)$ \\
\hline \multirow[t]{2}{*}{$d_{t-4}$} & -0.036 & -0.036 & -0.035 & -0.035 & -0.022 \\
\hline & $(-10.6)$ & $(-9.9)$ & $(-9.5)$ & $(-10.2)$ & $(-13.0)$ \\
\hline \multirow{2}{*}{$\theta_{t-1}$} & 0.955 & 0.954 & 0.956 & 0.955 & 0.989 \\
\hline & (191.9) & $(192.2)$ & $(182.6)$ & $(226.1)$ & $(499.4)$ \\
\hline \multirow[t]{2}{*}{ Volume change } & - & - & -0.0032 & -0.0032 & -0.071 \\
\hline & & & $(-5.7)$ & $(-5.0)$ & $(-10.1)$ \\
\hline \multirow[t]{2}{*}{ Trade intensity change } & - & - & -0.0020 & -0.0020 & 0.00001 \\
\hline & & & $(-4.7)$ & $(-5.2)$ & $(0.2)$ \\
\hline \multirow[t]{2}{*}{ Spread change } & - & - & -0.488 & -0.487 & -0.374 \\
\hline & & & $(-8.5)$ & $(-8.7)$ & $(-10.1)$ \\
\hline \multirow[t]{2}{*}{$\delta_{0}$} & - & -0.00007 & - & -0.00007 & 0.0001 \\
\hline & & $(-0.4)$ & & $(-0.3)$ & $(1.4)$ \\
\hline \multirow[t]{2}{*}{$\delta_{1}$} & - & 0.00032 & - & 0.00034 & 0.00003 \\
\hline & & (1.1) & & $(1.2)$ & $(0.2)$ \\
\hline \multirow[t]{2}{*}{$\delta_{2}$} & - & -0.00001 & - & 0.00003 & -0.00005 \\
\hline & & $(-0.1)$ & & $(0.1)$ & $(-0.7)$ \\
\hline \multirow[t]{2}{*}{ Constant } & -0.039 & -1.039 & -0.036 & -1.036 & -1.008 \\
\hline & $(-10.0)$ & $(-267.5)$ & $(-10.2)$ & $(-340.1)$ & $(-771.4)$ \\
\hline $\mathrm{LB}_{100}$ & 514.9 & 514.2 & 512.3 & 511.0 & 331.3 \\
\hline $\mathrm{LB}_{100}^{2}$ & 27.2 & 27.7 & 23.4 & 23.8 & 40.5 \\
\hline $\ln L$ & -138690.8 & -138588.0 & -138265.9 & -138262.5 & - \\
\hline
\end{tabular}

Note: $\mathrm{LB}_{100}$ is the Ljung-Box statistic of the residuals over 100 lags. $\mathrm{LB}_{100}^{2}$ is the same statistic for squared residuals. Residuals are calculated as $\left(d_{N^{k}(t)}^{k}-\widehat{\theta}_{N^{k}(t)}^{k}\right) / \widehat{\theta}_{N^{k}(t)}^{k}$ 
Table 3: Estimates of AstraZeneca models with explanatory variables and durations from Ericsson B (robust $t$ statistics in parenthesis).

\begin{tabular}{|c|c|c|c|c|c|}
\hline Variable & Model 1 & Model 2 & Model 3 & Model 4 & $\overline{\text { CLS }}$ \\
\hline \multirow[t]{2}{*}{$d_{t-1}$} & 0.073 & 0.073 & 0.082 & 0.082 & 0.040 \\
\hline & $(10.6)$ & $(9.7)$ & $(12.8)$ & $(13.4)$ & $(6.2)$ \\
\hline \multirow[t]{2}{*}{$d_{t-2}$} & -0.019 & -0.020 & -0.025 & -0.026 & -0.010 \\
\hline & $(-1.9)$ & $(-1.9)$ & $(-2.7)$ & $(-2.8)$ & $(-0.9)$ \\
\hline \multirow[t]{2}{*}{$d_{t-3}$} & -0.014 & -0.014 & -0.013 & -0.013 & -0.006 \\
\hline & $(-1.4)$ & $(-1.3)$ & $(-1.3)$ & $(-1.3)$ & $(-0.7)$ \\
\hline \multirow[t]{2}{*}{$d_{t-4}$} & 0.010 & 0.010 & 0.004 & 0.004 & 0.013 \\
\hline & $(1.0)$ & $(1.1)$ & $(0.3)$ & $(0.4)$ & $(1.4)$ \\
\hline \multirow[t]{2}{*}{$d_{t-5}$} & -0.029 & -0.028 & -0.028 & -0.027 & -0.026 \\
\hline & $(-4.0)$ & $(-3.7)$ & $(-3.3)$ & $(-3.6)$ & $(-3.3)$ \\
\hline \multirow[t]{2}{*}{$\theta_{t-1}$} & 0.974 & 0.973 & 0.976 & 0.975 & 0.981 \\
\hline & $(222.8)$ & $(145.4)$ & $(195.3)$ & $(154.2)$ & $(87.4)$ \\
\hline \multirow[t]{2}{*}{ Volume change } & - & - & -0.019 & -0.001 & -0.082 \\
\hline & & & $(-3.1)$ & $(-2.0)$ & $(-5.1)$ \\
\hline \multirow[t]{2}{*}{ Trade intensity change } & - & - & -0.001 & -0.019 & -0.002 \\
\hline & & & $(-2.1)$ & $(-2.5)$ & $(-1.6)$ \\
\hline \multirow[t]{2}{*}{ Spread change } & - & - & 0.658 & 0.659 & 0.404 \\
\hline & & & $(25.4)$ & $(25.2)$ & (19.0) \\
\hline \multirow[t]{2}{*}{$\delta_{0}$} & - & -0.00022 & - & -0.00023 & -0.00001 \\
\hline & & $(-0.6)$ & & $(-0.6)$ & $(-0.03)$ \\
\hline \multirow[t]{2}{*}{$\delta_{1}$} & - & 0.0011 & - & 0.00028 & 0.00085 \\
\hline & & $(0.8)$ & & $(0.2)$ & $(0.6)$ \\
\hline \multirow[t]{2}{*}{$\delta_{2}$} & - & 0.0015 & - & 0.0020 & 0.0001 \\
\hline & & $(0.9)$ & & (1.1) & $(0.07)$ \\
\hline \multirow[t]{2}{*}{ Constant } & -0.023 & -1.025 & -0.021 & -1.024 & -1.011 \\
\hline & $(-6.3)$ & $(-183.9)$ & $(-5.0)$ & $(-179.8)$ & $(-162.9)$ \\
\hline $\mathrm{LB}_{100}$ & 103.5 & 104.7 & 127.2 & 125.7 & 227.4 \\
\hline $\mathrm{LB}_{100}^{2}$ & 6.4 & 6.5 & 18.7 & 18.2 & 3.7 \\
\hline $\ln L$ & -16014.4 & -16005.7 & -15326.3 & -15318.7 & - \\
\hline
\end{tabular}

Note: $\mathrm{LB}_{100}$ is the Ljung-Box statistic of the residuals over 100 lags. $\mathrm{LB}_{100}^{2}$ is the same statistic for squared residuals. Residuals are calculated as $\left(d_{N^{k}(t)}^{k}-\widehat{\theta}_{N^{k}(t)}^{k}\right) / \widehat{\theta}_{N^{k}(t)}^{k}$. 
Granger causing Ericsson B, while Ericsson B Granger causes AstraZeneca. The result may be explained by the dominance of Ericsson B on the Stockholm Stock Exchange. Similar results have also been found for other stocks and markets, e.g., Spierdijk, et al. (2002) and Bauwens and Hautsch (2004).

In the models reported in Tables 2-3 there is serial correlation left that we were unable to reduce further. Figure 2 shows the serial correlation patterns for Model 4 (Table 2) and Model 4 (Table 3). The serial correlations are rather small and are decreasing at a slow rate for both stocks. To test for remaining serial correlation the Ljung-Box statistic is used. The test statistic has asymptotically $\chi_{100}^{2}$ distribution with 5 percent critical value of 124.3 . For the residuals of Ericsson B the null of no serial correlation is rejected for all models. For AstraZeneca the null hypothesis is rejected when spread, volume and trade intensity change is included in the model. For the squared residuals the null hypothesis is never rejected. Note that the reported results are based on deseasonalized data. The serial correlations becomes slightly smaller when data are deseasonalized. The omission of insignificant parameters, the third and fourth lags of durations for AstraZeneca, only result in small changes of the Ljung-Box statistics.
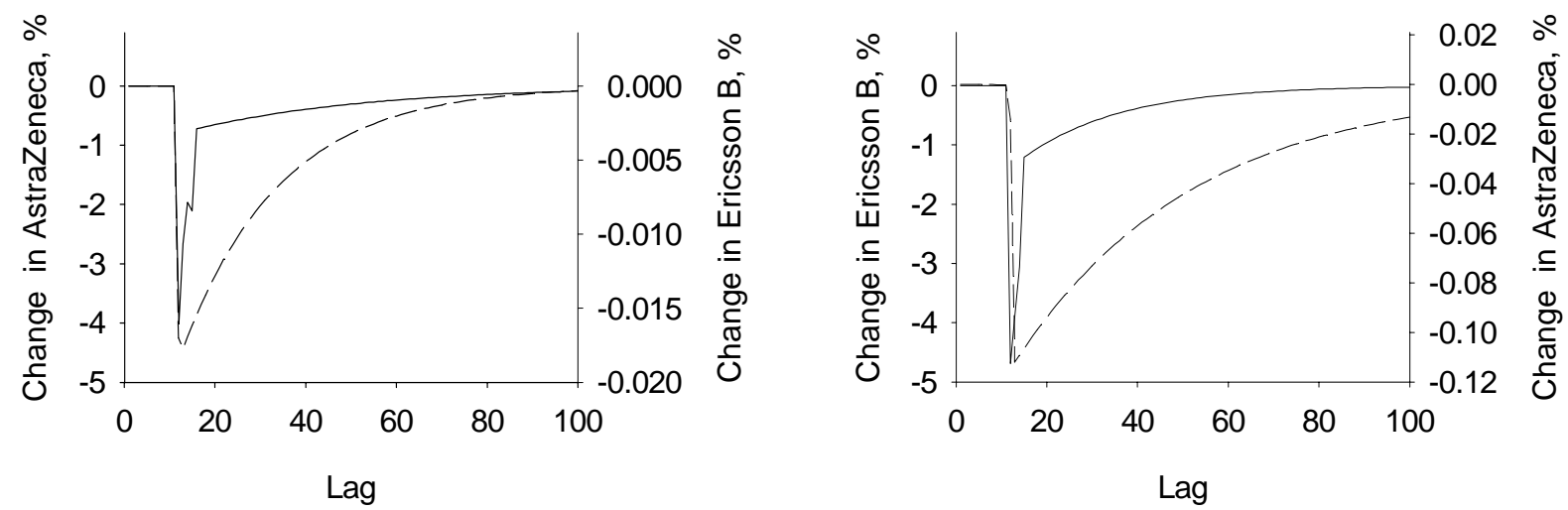

Figure 4: The change in percent of a 50 percent shortened duration from the mean duration at time $t=10$ in AstraZeneca (left) and Ericsson B (right). The solid line in the left figure shows the response in AstraZeneca of a shortened duration in AstraZeneca and in the right figure the response in Ericsson B of a shortened duration in Ericsson B. The dashed lines show the responses of a shorthened duration in the other stock.

The estimated models may be used to evaluate and illustrate the response to a chock in, e.g., the added duration. In Figure 4 the response in the expected mean duration $\theta_{N^{k}(t)}^{k}$ to a 50 percent reduction in the durations for AstraZeneca and Ericsson B is illustrated. The shocks have a small and diminishing effect on $\theta_{N^{k}(t)}^{k}$. Hence, the estimated models are stationary. The response in AstraZeneca of a shortened duration in AstraZeneca and the response in Ericsson 
B of a shortened duration in Ericsson B are of about the same size. The response in percent in AstraZeneca of a shortened duration in Ericsson is larger than the response in Ericsson B of a shortened duration in AstraZeneca, however, it is not very large.

\section{Conclusions}

The paper proposed adding durations from a second stock to the conditional mean function in the ACD model of Engle and Russell (1998). By including durations from a second stock dependence between duration series is captured in the model.

In the empirical part, we find that Ericsson B is Granger causing AstraZeneca, but not vice versa. This result is what may be expected with respect to the size of Ericsson B on the Stockholm Stock Exchange. In view of this empirical result the suggested model extension is able of capturing dependence between duration series and of providing an improvement in the econometric specification of the model. The attraction of the extension of the model is the simplicity in which information from other duration series can be added to the ACD model.

The parameter estimates of volume, spread and trade intensity changes are all significant for both AstraZeneca and Ericsson B. The sign of the parameters are negative for all but the spread change in AstraZeneca. The descriptive statistics of duration and other variables show presence of time of day seasonality in the stocks from the Stockholm Stock Exchange, cf. the results for other stock markets. 


\section{References}

Bauwens, L., F. Galli, and Giot, P. (2002). The moments of log-ACD models. unpublished manuscript, CORE, Louvain-La-Neuve.

Bauwens, L., Giot, P. (2000). The logarithmic ACD Model: An application to the Bid/Ask quote process of two NYSE stock. Annales d'Economie et de Statistique, 60, 117-149.

Bauwens, L., Giot, P. (2001). Econometric Modelling of Stock Market Intraday Activity. Kluwer, Dordrecht.

Bauwens, L., Giot, P., Grammig, J., Veredas, D., (2004). A comparison of financial duration models via density forecast. International Journal of Forecasting, 20, 589-609.

Bauwens, L., Hautsch, N. (2004) Dynamic Latent Factor Models for Intensity Processes. Core Discussion Paper 2003/103.

Brännäs, K., Simonsen, O. (2003). Discretized time and conditional duration modelling for stock transaction data. Umeå Economic Studies, 610.

Darolles, S., Gouriéroux, C. (2000). Intraday Transaction Price Dynamics. Annales d'Economie et de Statistique, 60, 207-237.

Easley, D,. O'Hara, M. (1992), Time and the process of security price adjustment. Journal of Finance, 47, 577-606.

Engle, R.F., Lunde. (2003). Trades and Quotes: A Bivariate Point Process. Journal of Financial Econometrics, 2, 159-188.

Engle, R.F., Russell, J.R. (1998). Autoregressive Conditional duration: A New Model for Irregularly Spaced Transaction Data. Econometrica, 66, 1127-1162.

Grammig, J., Wellner, M. (2002). Modeling the interdependence of volatility and intertransaction duration processes. Journal of Econometrics, 106, 369-400.

Granger, C. (1969). Investigating Causal Relations by Econometric Models and Crossspectral Methods. Econometrica, 37, 424-438.

Hansen, L.P., (1982). Large sample properties of generalized method of moments estimators. Econometrica, 50, 1029-1054.

O'Hara, M. (1994). Market Microstructure Theory. Blackwell Publishers Ltd.

Spierdijk, L., Nijman, T.E. and van Soest, A.H.O. (2002). Modeling Comovements in Trading Intensities to Distinguish Sector and Stock Specific News. Unpublished manuscript, Tilburg University.

Russell, J.R. (1999). Econometric Modelling of Multivariate Irregulary-Spaced High-Frequency Data. Unpublished manuscript, University of Chicago. 